\title{
Focus on adults with congenital heart disease: a growing issue
}

\author{
Douglas S Moodie
}

More than a million people in the US have congenital heart disease (CHD). In fact, in the US the population of patients with CHD comprises a greater number of patients who are 20 years or older than patients who are younger than 20 years. Since the days when infants with CHD needed immediate surgery to survive the first few months of life, there have been huge advances in the management of patients with congenital cardiac defects and, therefore, a huge change in the congenital heart population. Now, most infants with CHD survive well into adulthood. With modern surgical techniques and the availability of sophisticated diagnostic methods enabling even earlier diagnosis, $85 \%$ of the 25,000 infants born each year with CHD will reach adulthood.

As these patients become adults with congenital cardiac defects, they face many issues. The issue of aging itself and the high risk of developing arrhythmia seen in many of these patients means that they require constant attention and evaluation. The overall function of the heart also needs regular assessment, particularly as it relates to the development of congestive heart failure and the possible need for transplantation.

The problem faced in the US and Europe today is that patients with CHD continue to 'fall through the cracks' of our medical systems. Pediatric cardiologists frequently stop seeing patients after the age of 16-18 years because their practices are focused in dedicated children's hospitals, which cannot provide care for middle-aged adults. Adult cardiologists, on the other hand, are in general poorly trained in CHD and associated issues, and do not understand the daily management and follow-up these patients require.

Adult patients with CHD can be well cared for in the US, but there are only a few centers that can provide the needed expertise. Consequently, many patients have to travel considerable distances to receive the type and

\section{The problem \\ faced in the \\ US and Europe \\ today is that \\ patients with \\ CHD continue \\ to 'fall through \\ the cracks' of \\ our medical \\ systems.}

DS Moodie is an

Advisory Board

Member of Nature

Clinical Practice

Cardiovascular

Medicine.

\section{Competing interests}

The author declared no

competing interests.

www.nature.com/clinicalpractice doi:10.1038/ncpcardio1293 quality of care they need in order to maintain a good quality of life. In these specialized centers, care of the adult patient with CHD is a coordinated effort between pediatric and adult cardiology practitioners; however, many patients will also receive care from clinicians with expertise in such diverse disciplines as electrophysiology, treatment of heart failure, transplantation, hematology/oncology, nephrology, infectious disease, and genetics.

A smooth 'transition' from pediatric cardiology to adult cardiology for adults with $\mathrm{CHD}$ is extremely important but difficult to accomplish in most centers. We need a coordinated effort in our major centers between the pediatric cardiologists dealing with young CHD patients and whoever manages these patients as they age. The details of the effort are unimportant, as long as patients receive consistent care throughout their life; the many centers in the country that achieve excellent results achieve them in very different ways.

This issue of Nature Clinical Practice Cardiovascular Medicine includes important manuscripts related to adult patients with $\mathrm{CHD}$, covering issues ranging from long-term follow-up and arrhythmia, to the presentation of congenital cardiac defects in adulthood. The management of adult $\mathrm{CHD}$ remains a challenge, and so a special focus issue of this journal is important because it not only provides information to cardiologists and cardiac surgeons but also highlights the ongoing need for improvement in adult CHD care. Only with the dissemination of knowledge such as that presented within this focus issue can we improve our management of these patients. Much of the field of adult CHD is complex and requires the integration and cooperation of many disciplines to provide optimum care. The more knowledge disseminated through the medical literature, the more our diagnosis, management and long-term follow-up of these patients will improve. 